\title{
Oral conditions and dysphagia in Japanese, community-dwelling middle- and older- aged adults, independent in daily living
}

This article was published in the following Dove Press journal:

Clinical Interventions in Aging

I4 March 2017

Number of times this article has been viewed

\author{
Akinari Inui ${ }^{1}$ \\ Ippei Takahashi \\ Sizuka Kurauchi \\ Yuki Soma ${ }^{2}$ \\ Toshiaki Oyama' \\ Yoshihiro Tamura' \\ Takao Noguchi' \\ Kouichi Murashita ${ }^{3}$ \\ Shigeyuki Nakaji ${ }^{2}$ \\ Wataru Kobayashi' \\ 'Department of Oral and Maxillofacial \\ Surgery, ${ }^{2}$ Department of Social \\ Medicine, ${ }^{3} \mathrm{COI}$ Research Initiatives \\ Organization, Hirosaki University \\ Graduate School of Medicine, \\ Hirosaki, Aomori Prefecture, Japan
}

Correspondence: Akinari Inui Department of Oral and Maxillofacial Surgery, Hirosaki University Graduate School of Medicine, 5 Zaifutyo, Hirosaki 036-8562, Aomori Prefecture, Japan

$\mathrm{Tel}+8 \mathrm{I} I 72395127$

Fax $+8 \mid 172395128$

Email hI4gm308@hirosaki-u.ac.jp
Purpose: Prevention, early detection and effective rehabilitation of dysphagia are important issues to be considered in an aging society. Previous studies have shown conflicting findings regarding the association between dysphagia and its potential risk factors, including age, malnutrition, oral conditions, lifestyle and medical history. Herein, we assessed the prevalence and association of dysphagia with potential risk factors in 50- to 79-year-old adults dwelling in a community in Japan.

Patients and methods: In this study, there were 532 participants (185 males and 347 females). Participants who responded positively to the question "Do you sometimes choke on drinks/food such as tea and soup?" or those who presented with abnormal repetitive saliva swallowing test findings were diagnosed with dysphagia. The data collected from these participants included the following: number of teeth, occurrence of oral dryness, age, body mass index, serum albumin concentration, smoking, drinking and exercise habits, presence of diseases, such as diabetes mellitus and hypertension, and questions from the Mini-Mental State Examination.

Results: Dysphagia was observed in 33 males (17.8\%) and 76 females (21.9\%). To explore the effect of the potential risk factors on the prevalence of dysphagia, a model was built by multivariate logistic regression analysis. Using the forced entry method, oral dryness (odds ratio $[\mathrm{OR}]=3.683$ and $P=0.003$ in males; $\mathrm{OR}=1.797$ and $P=0.032$ in females) and the number of teeth ( $\mathrm{OR}=0.946$ and $P=0.038$ in males) were found to be significantly related to dysphagia.

Conclusion: This cross-sectional study demonstrated associations between oral conditions and dysphagia. Factors such as oral dryness and number of teeth may contribute to dysphagia more so than aging, lifestyle and comorbidity in community-dwelling adults over the age of 50 .

Keywords: oral dryness, number of teeth, swallow, nutrition, lifestyle

\section{Introduction}

Swallowing is an essential part of life; disruptions in swallowing affect the quality of life, which is a major concern in an aging society. Dysphagia or swallowing impairment refers to difficulty or discomfort during the progression of the alimentary bolus from the oral cavity to the stomach. ${ }^{1}$ It is strongly associated with aspiration pneumonia in older people and has been shown to result in extended hospital stay, worsening of other diseases and increased risk of death in the elderly. Therefore, prevention, early detection and effective rehabilitation of dysphagia are important issues to improve health in the elderly. ${ }^{2}$ However, only a few epidemiological studies have reported the prevalence of dysphagia in community-dwelling populations. Previously, Serra-Prat et al reported that the signs of dysphagia were prevalent in $27.2 \%$ of subjects aged 70 and older. ${ }^{3}$ Additionally, previous studies have shown conflicting findings regarding 
the association between dysphagia and potential risk factors, such as age, malnutrition, oral conditions, lifestyle and medical history (such as cognitive function). ${ }^{2-7}$ These studies included participants with various characteristics, such as pneumonia patients, residents in care homes, frail elderly people and the community-dwelling population. Okamoto et al reported the relationship between dysphagia and tooth loss in community-dwelling independent elderly adults. ${ }^{4}$ Another study showed that Care Dependency Scale scores, malnutrition and disease clusters, such as dementia, nervous system disorders and cerebrovascular disease/hemiparesis, were associated with the presence of subjective dysphagia. ${ }^{5}$ Lindgren and Janzon reported complaints of swallowing difficulty with several clinical findings among 50- to 79-year-old males and females living in an urban area; they described the consequences of these symptoms in terms of changing dietary habits and medical history but were unable to define potential risk factors of dysphagia., ${ }^{4,6}$ In previous studies, the number of examinations for participants was different. Furthermore, risk factors were not examined by sex differences. Therefore, previous studies have not been able to clearly indicate potential risk factors for dysphagia.

Changes in physical performance, oral conditions and medical history generally begin at 50 years of age,${ }^{6}$ and loss of skeletal muscle mass is prominent after this age in Japanese adults. ${ }^{8}$ Habitual exercise has been shown to be effective in maintaining physical performance in the elderly. ${ }^{9,10}$ The National Dental Survey conducted in Japan revealed that the prevalence rates of periodontitis in those aged $40-45$ and $55-59$ years were $25.6 \%$ and $47.0 \%$, respectively. ${ }^{11}$ Several epidemiological studies have identified potential risk factors of dysphagia in community-dwelling older adults. Epidemiological studies evaluating the prevention, early detection and effective rehabilitation of dysphagia should focus on patients above 50 years of age. Thus, the present study aimed to examine the frequency of dysphagia and its risk factors in rural, community-dwelling people aged 50-80 years.

\section{Patients and methods}

\section{Participants}

This study was a part of an ongoing community-based project entitled the Iwaki Health Promotion Project and comprised 651 volunteers (234 males and 417 females; $\geq 50$ years) who participated in 2015. These participants were rural residents of the Iwaki district located in Hirosaki in northern Japan. The overall objective of this project was health promotion for the general population. All study subjects participated voluntarily in the Iwaki project in response to a public announcement, and it was estimated that more than 1,000 males and females participated in this study. They participated cognitively and were physically capable of reaching the research venue by referring to a map. The exclusion criteria in the present study were medical histories of cancer, stroke, ischemic heart disease and Parkinson's disease. Additionally, patients with incomplete data were excluded from the study; in total, 532 participants (185 males and 347 females) were included. Data collection for this study was approved by the Ethics Committee of the Hirosaki University School of Medicine, and all participants provided written informed consent.

Demographic data, such as age and sex; lifestyle, such as smoking and drinking; and medical history were obtained from self-reported questionnaires and interviews. To assess the exercise habits of the participants, they were also asked to choose from two items: none or 1-7 days/week. The height and weight of the participants were measured, and body mass index (BMI) was calculated using the following equation: BMI $\left(\mathrm{kg} / \mathrm{m}^{2}\right)=$ weight $(\mathrm{kg}) /($ height $[\mathrm{m}] \times$ height $[\mathrm{m}])$. Malnutrition was assessed based on BMI $<20 \mathrm{~kg} / \mathrm{m}^{2}$. In this study, serum albumin (ALB) concentration $(\mathrm{g} / \mathrm{dL})$ was considered as a confounding factor because it represents nourishment. Non-fasting blood samples were obtained, and plasma concentrations of ALB were measured using standard procedures in conjunction with a completely automated system (SRL Ltd, Tokyo, Japan). The Mini-Mental State Examination (MMSE) was administered to all participants to measure their global cognitive status. This test assessed the orientation to place and time, short-term memory, episodic long-term memory, subtraction ability, ability to construct sentences and oral language ability. The maximum score was set as $30 .{ }^{12}$

\section{Assessment of dysphagia}

To evaluate subjective dysphagia, we asked the question "Do you sometimes choke on drinks/food such as tea and soup?". An objective assessment of dysphagia was carried out using the repetitive saliva swallowing test (RSST), which detects patients who experience aspiration. ${ }^{13,14}$ The patients were asked to perform saliva swallows (dry) as many times as possible in 30 seconds. If the patient was unable to perform three consecutive swallows, he or she was likely to have dysphagia associated with aspiration. Participants who responded positively to the question or presented with abnormal RSST findings were diagnosed with impaired swallowing.

\section{Oral examination}

Dental examinations were conducted by trained and experienced dentists under artificial lighting, with both the dentist 
and the patient in seated positions. The teeth present were defined as healthy, carious or treated. The participants were asked as to whether they had subjective oral dryness.

\section{Statistical analysis}

Differences between groups were determined using Student's $t$-test and chi-square test. Additionally, odds ratios (ORs) with $95 \%$ confidence interval $(95 \% \mathrm{CI})$ were calculated using multiple logistic regression analysis. To explore the effect of potential risk factors of dysphagia on the prevalence of subjective dysphagia, a model was built by multivariate analysis using the forced entry method. Variables presenting a $P$-value of $<0.25$ in the univariate analysis were then included in the multivariate model as non-cases (0) and cases (1) of dysphagia. Goodness of fit was performed based on the technique of Hosmer and Lemeshow. A $P$-value of $<0.05$ was applied as the cutoff. The Hosmer and Lemeshow value was calculated to indicate the fit of the final model: the higher the $P$-value, the better the fit of the model. Statistical Package for the Social Sciences (SPSS) for Windows (Version 24; IBM Corp., Armonk, NY, USA) was used for statistical analysis.

\section{Results}

\section{Prevalence of dysphagia}

Dysphagia was observed in 33 males (17.8\%), 76 females (21.9\%) and a combination of 109 males and females (20.5\%) of the 532 participants, as shown in Figures 1 and 2, respectively. With respect to age, the highest prevalence was seen in 70- to 74-year-old males $(40.0 \%)$ and in 75 - to 79-yearold females $(41.4 \%)$, and the lowest prevalence was seen in

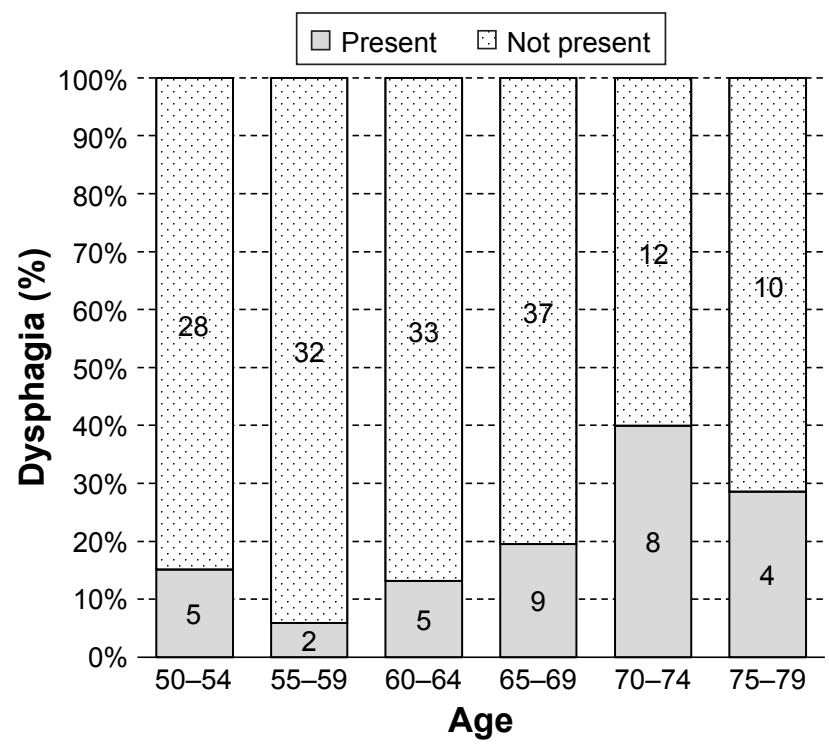

Figure I The age and associated dysphagia of community-dwelling older adults (males).

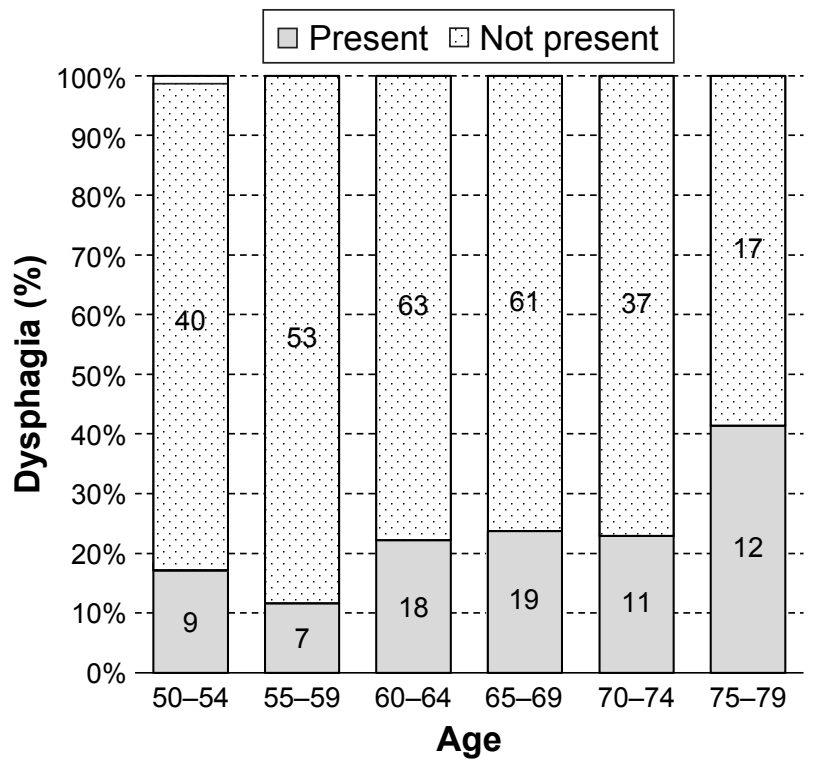

Figure 2 The age and associated dysphagia of community-dwelling older adults (females).

both 55- to 59-year-old males and females (5.9\% and $11.7 \%$, respectively).

\section{Univariate analysis}

In the univariate analysis, depending on the presence or absence of dysphagia (Tables 1 and 2), significant differences in the number of teeth $(P=0.014$ in males $)$, oral dryness

Table I Characteristics of community-dwelling older adults (males) with and without dysphagia

\begin{tabular}{|c|c|c|c|}
\hline \multirow[t]{2}{*}{ Variables } & \multirow{2}{*}{$\frac{\text { Not present }}{(n=\mid 52)}$} & \multirow{2}{*}{$\frac{\text { Present }}{(n=33)}$} & \multirow[t]{2}{*}{$P$-value } \\
\hline & & & \\
\hline Number of teeth & $23.8 \pm 7.3$ & $19.3 \pm 9.7$ & 0.014 \\
\hline Oral dryness & 39 (25.7\%) & 17 (5I.5\%) & 0.003 \\
\hline Age & $61.9 \pm 7.1$ & $65.5 \pm 7.7$ & 0.014 \\
\hline $\mathrm{BMI}<20.0 \mathrm{~kg} / \mathrm{m}^{2}$ & $13(8.6 \%)$ & $3(9.1 \%)$ & 0.921 \\
\hline ALB & $4.5 \pm 0.3$ & $4.4 \pm 0.3$ & 0.122 \\
\hline Smoking habit & & & 0.971 \\
\hline None & 60 (39.5\%) & 14 (42.4\%) & \\
\hline Current & $36(23.7 \%)$ & $6(18.2 \%)$ & \\
\hline Previous & $56(36.8 \%)$ & $13(39.4 \%)$ & \\
\hline Drinking habit & & & 0.344 \\
\hline None & 34 (22.4\%) & 9 (27.3\%) & \\
\hline Current & $112(73.7 \%)$ & $21(63.6 \%)$ & \\
\hline Previous & $6(3.9 \%)$ & $3(9.1 \%)$ & \\
\hline Exercise habit & & & 0.250 \\
\hline$\geq$ I day/week & 32 (21.1\%) & $10(30.3 \%)$ & \\
\hline Diabetes mellitus & $13(8.6 \%)$ & $5(15.2 \%)$ & 0.246 \\
\hline Hypertension & $60(39.5 \%)$ & $15(45.5 \%)$ & 0.526 \\
\hline MMSE & $29.0 \pm 1.5$ & $28.8 \pm 2.1$ & 0.873 \\
\hline
\end{tabular}

Notes: Student's t-test or chi-square test. Values are presented as mean \pm SD or $n(\%)$.

Abbreviations: ALB, albumin; BMI, body mass index; MMSE, Mini-Mental State Examination; SD, standard deviation. 
Table 2 Characteristics of community-dwelling older adults (females) with and without dysphagia

\begin{tabular}{|c|c|c|c|}
\hline \multirow[t]{2}{*}{ Variables } & \multirow{2}{*}{$\frac{\text { Not present }}{(n=27 I)}$} & \multirow{2}{*}{$\begin{array}{l}\text { Present } \\
(n=76)\end{array}$} & \multirow[t]{2}{*}{$P$-value } \\
\hline & & & \\
\hline Number of teeth & $21.1 \pm 8.5$ & $19.8 \pm 9.7$ & 0.373 \\
\hline Oral dryness & 106 (39.1\%) & $43(56.6 \%)$ & 0.007 \\
\hline Age & $62.8 \pm 7.3$ & $65.2 \pm 7.6$ & 0.015 \\
\hline $\mathrm{BMI}<20.0\left(\mathrm{~kg} / \mathrm{m}^{2}\right)$ & $64(23.6 \%)$ & 12 (I5.8\%) & 0.101 \\
\hline ALB & $4.5 \pm 0.3$ & $4.4 \pm 0.3$ & 0.114 \\
\hline Smoking habit & & & 0.171 \\
\hline None & 226 (83.4\%) & $66(86.8 \%)$ & \\
\hline Current & 17 (6.3\%) & 7 (9.2\%) & \\
\hline Previous & $28(10.3 \%)$ & $3(3.9 \%)$ & \\
\hline Drinking habit & & & 0.889 \\
\hline None & $192(70.8 \%)$ & $56(73.7 \%)$ & \\
\hline Current & $7 \mathrm{I}(26.2 \%)$ & $18(23.7 \%)$ & \\
\hline Previous & $8(3.0 \%)$ & $2(2.6 \%)$ & \\
\hline Exercise habit & & & 0.059 \\
\hline$\geq$ I day/week & $70(25.8 \%)$ & 28 (36.8\%) & \\
\hline Diabetes mellitus & II (4.I\%) & $4(5.3 \%)$ & 0.648 \\
\hline Hypertension & $96(35.4 \%)$ & $35(46.1 \%)$ & 0.091 \\
\hline MMSE & $29.2 \pm 1.3$ & $29.0 \pm 1.8$ & 0.301 \\
\hline
\end{tabular}

Notes: Student's t-test or chi-square test. Values are presented as mean \pm SD or $\mathrm{n}(\%)$. Abbreviations: ALB, albumin; BMI, body mass index; MMSE, Mini-Mental State Examination; SD, standard deviation.

( $P=0.003$ in males, $P=0.007$ in females) and age $(P=0.014$ in males, $P=0.015$ in females) were noted. The average number of teeth in males without dysphagia was $23.8 \pm 7.3$ and in males with dysphagia was $19.3 \pm 9.7$. The number of participants without dysphagia and oral dryness included 39 males (25.7\%) and 106 females (39.1\%); the number of participants with dysphagia and oral dryness was 17 males $(51.5 \%)$ and 43 females $(56.6 \%)$.

\section{Multiple logistic regression analysis}

Multiple logistic regression analysis was performed, where the dependent variable was the presence or absence of dysphagia and the independent variable includes the number of teeth, oral dryness, age, BMI $<20.0 \mathrm{~kg} / \mathrm{m}^{2}$, serum ALB concentration, smoking habits, exercise habits, diabetes mellitus status and hypertension status. Logistic regression analysis using the forced entry method showed statistical significance for oral dryness in males and females $(P=0.003$ and 0.032 , respectively; Table 3). In men, the ORs for oral dryness and number of teeth were 3.683 and 0.946 (95\% CI $=1.570-8.644$ and 0.898-0.997, respectively). This model was shown to be compatible with the Hosmer-Lemeshow test $(P=0.109)$, with a predictive accuracy of $83.8 \%$. In females, the OR for oral dryness was $1.804(95 \% \mathrm{CI}=1.054-3.089)$. This model was also shown to be compatible with the Hosmer-Lemeshow test $(P=0.526)$, with a predictive accuracy of $78.7 \%$.
Table 3 Logistic regression models for dysphagia using the forced entry method

\begin{tabular}{|c|c|c|c|}
\hline Variables & OR & $95 \% \mathrm{Cl}$ & $P$-value \\
\hline \multicolumn{4}{|l|}{ Males } \\
\hline Number of teeth & 0.946 & $0.898-0.997$ & 0.038 \\
\hline Oral dryness & 3.683 & $1.570-8.644$ & 0.003 \\
\hline Age & 1.041 & $0.972-1.115$ & 0.248 \\
\hline $\mathrm{BMI}<20.0 \mathrm{~kg} / \mathrm{m}^{2}$ & 0.856 & $0.198-3.707$ & 0.836 \\
\hline ALB & 0.526 & $0.114-2.423$ & 0.410 \\
\hline \multicolumn{4}{|l|}{ Smoking habit } \\
\hline None & I & & 0.445 \\
\hline Previous & 1.956 & $0.594-6.422$ & 0.270 \\
\hline Current & 2.064 & $0.639-6.663$ & 0.226 \\
\hline Exercise habit & $\mathrm{I} .477$ & $0.558-3.910$ & 0.432 \\
\hline Diabetes mellitus & 1.684 & $0.448-6.339$ & $0.44 I$ \\
\hline Hypertension & 0.678 & $0.266-1.725$ & 0.415 \\
\hline Constant & 0.345 & & 0.810 \\
\hline \multicolumn{4}{|l|}{ Females } \\
\hline Number of teeth & 1.000 & $0.967-1.035$ & 0.983 \\
\hline Oral dryness & 1.797 & $1.05 \mathrm{I}-3.070$ & 0.032 \\
\hline Age & 1.031 & $0.986-1.077$ & 0.186 \\
\hline $\mathrm{BMI}<20.0\left(\mathrm{~kg} / \mathrm{m}^{2}\right)$ & 0.622 & $0.306-1.264$ & 0.189 \\
\hline ALB & 0.599 & $0.233-1.543$ & 0.289 \\
\hline \multicolumn{4}{|l|}{ Smoking habit } \\
\hline None & I & & 0.163 \\
\hline Previous & 0.567 & $0.207-1.553$ & 0.270 \\
\hline Current & 0.224 & $0.048-1.044$ & 0.057 \\
\hline Exercise habit & 1.448 & $0.814-2.578$ & 0.208 \\
\hline Diabetes mellitus & 0.882 & $0.254-3.067$ & 0.844 \\
\hline Hypertension & 1.410 & $0.792-2.511$ & 0.243 \\
\hline Constant & 0.458 & & 0.781 \\
\hline
\end{tabular}

Notes: Males, Hosmer-Lemeshow analysis, $P=0.109$. Females, Hosmer-Lemeshow analysis, $P=0.526$.

Abbreviations: ALB, albumin; $\mathrm{BMI}$, body mass index; $\mathrm{Cl}$, confidence interval; OR, odds ratio.

\section{Discussion}

Several studies have examined the methods used to evaluate dysphagia. The use of video fluorescence or video endoscopy of swallowing has been recommended for precise examination of dysphagia in the clinical setting. ${ }^{5}$ However, these methods are considered impractical for investigating a community-dwelling population due to their complex apparatus and the need of radiation exposure. Thus, simple questionnaire and RSST were used to evaluate dysphagia in this study. One of the most common screening tests for dysphagia has shown a high correlation with video fluorography of swallowing, especially in the case of aspiration. ${ }^{14}$ The sensitivity and specificity of the RSST compared to the use of video fluorography were $98 \%$ and $66 \%$, respectively. In order to increase the accuracy of detection, a simple questionnaire was also used to ensure that certain conditions such as inapparent dysphagia were included in the result. ${ }^{5}$ The prevalence rates of dysphagia in our study were $17.8 \%$ in males and $21.9 \%$ in females. As these results were similar to those reported by 
Okamoto et al (15.1\%), this study was considered appropriate for the evaluation of dysphagia. ${ }^{4}$

In this study, there were significant differences between dysphagia and oral dryness in both sexes. ${ }^{4}$ Normally, crushed food is mixed with saliva to form bolus, thereby, enabling food to be swallowed easily. Oral dryness is affected by the preparation phase (chewing) of the feeding/ swallowing function. As dysphagia can result in reduced or altered oral intake of liquid, it increases the risk of oral dryness. ${ }^{3}$

In contrast to males, the number of teeth did not show any significant correlations with dysphagia in females. In some people with dysphagia, ingested food tends to remain in the mouth after a meal, as they are unable to facilitate the movement of the food from the oral cavity to the stomach by swallowing. As a result, their oral hygiene becomes poor, thereby, leading to loss of teeth.

The mechanism of swallowing is complex. It consists of occlusal support, mandibular stopping by occlusion, elevation of the hyoid bone, and movement of the larynx and tongue. The stability of the tongue apex has been shown to affect the swallowing process. ${ }^{15,16}$ When food is ingested, we use upper and lower teeth to crush the food. Thus, decrease in the number of remaining teeth reduces the fixation and stability of the tongue tip, which, in turn, affects the squeezing movement seen during swallowing. Age-related changes in the position of hyoid bone may be a component of decreased swallowing safety and aspiration in older adults. ${ }^{17}$ The distance between the hyoid bone and mandible increases with age, and the position of the hyoid bone is correlated with aspiration in older males. Such difference was distinct between males and females, and it was further affected by the number of teeth and prevalence of dysphagia in the present study. ${ }^{18}$

Considering the results obtained from this study, improvement in oral conditions might affect dysphagia. ${ }^{5,15,19}$ Other studies have shown that oral hygiene is related to dysphagia and aspiration pneumonia. It is important for patients with dysphagia to maintain oral hygiene, as it prevents tooth loss and reduces the risk of aspiration pneumonia by reducing the number of pathogens (such as bacteria implicated in periodontitis). ${ }^{20}$ Moreover, stimulations caused during oral care may affect the swallowing reflex and may improve swallowing ability. ${ }^{21}$ Furthermore, oral massage improves the flow rate of saliva in patients with xerostomia. ${ }^{22,23}$ Thus, management of oral condition might prove beneficial because of its association with the teeth health and other conditions, such as oral dryness, dysphagia and pneumonia. Although most of the studies investigated older or frail people, this study showed the relationship between oral conditions and dysphagia in middle-aged and elderly adults.

The deterioration of general condition and health habits may also lead to dysphagia. The physiology of swallowing changes with aging. ${ }^{2,7}$ Reductions in muscle mass and connective tissue and elasticity result in loss of strength and range of motion. van der Maarel-Wierink et al listed independent lifestyle, malnutrition, cognitive function, nervous system disease, cerebrovascular disease and hemiplegia as potential risk factors for swallowing disorders in elderly. ${ }^{5}$ Some researchers have also pointed out that nutritional status and muscle strength are related to motor function during swallowing. ${ }^{5,6,15}$ Furthermore, dementia patients are likely to be suffering with dysphagia due to impairment of the neurotransmission mechanism. ${ }^{2,7}$ In the current study, it was found that there was no significant difference in serum ALB concentration, BMI or exercise habits among the population, and age was not significantly related to dysphagia.

The results obtained from this study are not in accordance with those of previous studies. The reason for this may be due to the fact that unlike the previous studies, subjects who participated in this study were considered healthy. This is evident from the high MMSE scores $(28.8 \pm 2.1$ in men and 29.0 \pm 1.8 in women) obtained for current subjects. All study participants were cognitively and physically capable of arriving at the research venue by using a map. The results of this study are representative of dysphagia in the community-dwelling population or in the more healthy population compared to those of previous studies. Using the volume-viscosity swallow test, Serra-Prat et al have examined the severity of dysphagia. They reported that the signs of dysphagia, impaired swallowing efficacy, impaired safety and aspiration were prevalent in $27.2 \%, 20.5 \%, 15.4 \%$ and $6.7 \%$ of the samples, respectively. ${ }^{3}$ However, they did not examine oral conditions. In this study, we did not clarify the type of dysphagia, and we examined oral conditions. We speculated that oral dryness and the number of teeth might have stronger associations with swallowing efficacy (impaired labial seal, oral or pharyngeal residue and piecemeal deglutition) rather than swallowing safety (changes in voice quality, cough or decrease in oxygen saturation), especially because participants in this study were considered to be health conscious. Therefore, for general population or healthy population, oral dryness and the number of teeth were more critical factors than other factors, such as nutritional status, lifestyle or previous medical history. 


\section{Limitations}

There were several limitations to this study. As the study design was cross-sectional, it did not allow us to find out the detailed causal connections among the measured items. Thus, longitudinal studies must be considered in the future. Moreover, all subjects voluntarily participated in the Iwaki project in response to public announcement, and it was estimated that more than 1,000 males and females participated in this study. Therefore, the study participants were not randomly selected. Certain impaired efficacies (presence of oral, pharyngeal residue, piecemeal deglutition and impaired lip closure) are important part of dysphagia, although swallowing efficacy was not evaluated (only aspiration). The 10-item Eating Assessment Tool (EAT-10) that is a self-reported questionnaire or the symptoms associated with dysphagia (Sydney swallowing questionnaire) which assesses the severity of dysphagia. In this study, we did not examine the severity of dysphagia. However, the sensitivity and specificity of RSST compared to video fluorography were $98 \%$ and $66 \%$, respectively. RSST was found to be useful in the evaluation of dysphagia. Additionally, this study did not consider the effect of removable dentures. Hence, we were unable to consider the effect of dentures (denture position, form and suitability, etc.) on dysphagia. Future studies are warranted to dissect the impact of dentures, severity and type efficacy of dysphagia.

\section{Conclusion}

In this study, the prevalence of dysphagia was approximately $20 \%$ in a Japanese community-dwelling population aged 50-79 years. This cross-sectional study revealed relationships between oral conditions, especially oral dryness, and dysphagia. Thus, oral dryness and the number of teeth may be more important risk factors than aging, lifestyle and comorbidity when considering the dynamics of dysphagia in community-dwelling older adults. However, the interpretation of our results was hampered by lack of data, including insight about denture usage and longitudinal observations. Therefore, future comprehensive longitudinal studies are warranted.

\section{Acknowledgments}

The authors would like to thank all of their coworkers in this study (especially Kaori Sawada, Youhei Sekine, Rina Tanaka, Itoyo Tokuda, Anna Satake and Toshiyuki Osanai) for their contributions and assistance. This study was supported by grants-in-aid for Scientific Research (15K20847, $16 \mathrm{~K} 11879$ ) from the Japanese Ministry of Education, Culture, Sports, Science and Technology and by research aid from the Foundation Institute of Geriatric Medicine and Dentistry in Tokyo, 8020 Promotion Foundation in Tokyo and the Smoking Research Foundation in Tokyo. The authors would like to thank Enago (www.enago.jp) for the English language review.

\section{Author contributions}

AI conceived the study and wrote the initial draft of this article. IT designed the study, conducted the statistical analyses, interpreted the data and assisted in drafting the article. SY contributed to the study design and interpretation of the results and had full access to all of the data in the study. IT, SK and KS participated in the data collection. WK took responsibility for the integrity of the data and the accuracy of the data analysis. All authors have read and approved the final article. All authors contributed toward data analysis, drafting and critically revising the paper and agree to be accountable for all aspects of the work.

\section{Disclosure}

The authors report no conflicts of interest in this work.

\section{References}

1. Rofes L, Arreola V, Almirall J, et al. Diagnosis and management of oropharyngeal dysphagia and its nutritional and respiratory complications in the elderly. Gastroenterol Res Pract. 2011;2011:818979.

2. Sura L, Madhavan A, Carnaby G, Crary MA. Dysphagia in the elderly: management and nutritional considerations. Clin Interv Aging. 2012;7:287-298.

3. Serra-Prat M, Hinojosa G, López D, et al. Prevalence of oropharyngeal dysphagia and impaired safety and efficacy of swallow in independently living older persons. J Am Geriatr Society. 2011;59(1):186-187.

4. Okamoto N, Tomioka K, Saeki K, et al. Relationship between swallowing problems and tooth loss in community-dwelling independent elderly adults: the Fujiwara-Kyo study. J Am Geriatr Soc. 2012;60(5): 849-853.

5. van der Maarel-Wierink CD, Meijers JM, De Visschere LM, de Baat C, Halfens RJ, Schols JM. Subjective dysphagia in older care home residents: a cross-sectional, multi-centre point prevalence measurement. Int J Nurs Stud. 2014;51(6):875-881.

6. Lindgren S, Janzon L. Prevalence of swallowing complaints and clinical findings among 50-79-year-old men and women in an urban population. Dysphagia. 1991;6(4):187-192.

7. Park YH, Han HR, Oh BM, et al. Prevalence and associated factors of dysphagia in nursing home residents. Geriatr Nurs. 2013;34(3): 212-217.

8. Yamada M, Moriguch Y, Mitani T, Aoyama T, Arai H. Age-dependent changes in skeletal muscle mass and visceral fat area in Japanese adults from 40 to 79 years-of-age. Geriatr Gerontol Int. 2014;14 (Suppl 1): $8-14$.

9. Akune T, Muraki S, Oka H, et al. Exercise habits during middle age are associated with lower prevalence of sarcopenia: the ROAD study. Osteoporos Int. 2014;25(3):1081-1088.

10. Nishiguchi S, Yamada M, Kajiwara Y, et al. Effect of physical activity at midlife on skeletal muscle mass in old age in community-dwelling older women: a cross-sectional study. J Clin Gerontol Geriatr. 2014; $5(1): 18-22$. 
11. Ministry of Health Labour and Welfare. Statistical tables of the survey of dental diseases. Available from: http://www.mhlw.go.jp. Accessed January 15, 2017.

12. Folstein MF, Folstein SE, McHugh PR. "Mini-mental state". A practical method for grading the cognitive state of patients for the clinician. J Psychiatr Res. 1975;12(3):189-198.

13. Baba M, Saitoh E, Okada S. Dysphagia rehabilitation in Japan. Phys Med Rehabil Clin N Am. 2008;19(4):929-938, x.

14. Oguchi K, Saitoh E, Baba M, Kusudo S, Tanaka T, Onogi K. [The repetitive saliva wallowing test (RSST) as a screening test of functional dysphagia (2) validity of RSST]. Japanese J Rehabil Med. 2000;37(6): 383-388. Japanese.

15. Yoshikawa M, Yoshida M, Nagasaki T, et al. Aspects of swallowing in health dentate elderly persons older than 80 years. $J$ Gerontol A Biol Sci Med Sci. 2005;60(4):506-509.

16. Yoshikawa M, Yoshida M, Nagasaki T, Tanimoto K, Tsuga K, Akagawa Y. Influence of aging and denture use on liquid swallowing in healthy dentulous and edentulous older people. J Am Geriatr Soc. 2006; 54(3):444-449.

17. Feng X, Todd T, Hu Y, et al. Age-related changes of hyoid bone position in healthy older adults with aspiration. Laryngoscope. 2014;124(6): E231-E236.
18. Logemann JA, Pauloski BR, Rademaker AW, Kahrilas PJ. Oropharyngeal swallow in younger and older women: videofluoroscopic analysis. J Speech Lang Hear Res. 2002;45(3):434-445.

19. Yoneyama T, Yoshida M, Matsui T, Sasaki H. Oral care and pneumonia. Oral Care Working Group. Lancet. 1999;354(9177):515.

20. van der Maarel-Wierink CD, Vanobbergen JN, BronkhorstEM, Schols JM, de Baat C. Risk factors for aspiration pneumonia in frail older people: a systematic literature review. J Am Med Dir Assoc. 2011;12(5): 344-354.

21. Yoshino A, Ebihara T, Ebihara S, Fuji H, Sasaki H. Daily oral care and risk factors for pneumonia among elderly nursing home patients. JAMA. 2001;286(18):2235-2236.

22. Ohara Y, Yoshida N, Kono Y, et al. Effectiveness of an oral health educational program on community-dwelling older people with xerostomia. Geriatr Gerontol Int. 2015;15(4):481-489.

23. Kim EJ, Kwag JS. Change of salivary flow rate, xerostomia, and oral health-related quality of life after oral muscle massage. J Korean Soc Dent Hyg. 2015;15(4):679-685.
Clinical Interventions in Aging

\section{Publish your work in this journal}

Clinical Interventions in Aging is an international, peer-reviewed journal focusing on evidence-based reports on the value or lack thereof of treatments intended to prevent or delay the onset of maladaptive correlates of aging in human beings. This journal is indexed on PubMed Central, MedLine,

\section{Dovepress}

CAS, Scopus and the Elsevier Bibliographic databases. The manuscript management system is completely online and includes a very quick and fair peer-review system, which is all easy to use. Visit http://www.dovepress. com/testimonials.php to read real quotes from published authors. 